\title{
LETTER
}

Chronic lymphocytic leukemia

\section{Detection of chronic lymphocytic leukemia subpopulations in peripheral blood by phage ligands of tumor immunoglobulin B cell receptors}

\author{
Selena Mimmi $\mathbb{D}^{1}$ - Domenico Maisano $\mathbb{D}^{1} \cdot$ Nancy Nisticò $^{1} \cdot$ Eleonora Vecchio ${ }^{1}$. Federico Chiurazzi ${ }^{2} \cdot$ Katia Ferrara $^{2}$. \\ Marialuigia lannalfo ${ }^{2}$ - Alessandro $\mathrm{D}^{\prime}$ Ambrosio $^{2}$ - Giuseppe Fiume ${ }^{1}$ Enrico laccino ${ }^{1}$ - lleana Quinto $\mathbb{D}^{1}$
}

Received: 17 March 2020 / Revised: 15 May 2020 / Accepted: 21 May 2020 / Published online: 1 June 2020

(c) The Author(s) 2020. This article is published with open access

\section{To the Editor:}

The B cell receptor (BCR) is an immunoglobulin (Ig) expressed on the membrane surface of mature $B$ cells [1]. The IgBCR has two heavy and two light chains, each one made by a constant and a variable region. The variable sequences of the IgBCR are generated during B-cell differentiation through somatic recombination, so called VDJ recombination, and somatic hypermutation [2]. Once assembled on the B-cell surface, the IgBCR recognizes a specific antigen through its binding to the variable regions. This event triggers the B-cell immune response against the antigen. Since the variable regions are B cell specific, the sequence of IgBCR allows the identification of single B-cell clones [3]. In B-lymphoproliferative disorders, the IgBCR plays a key role in the development, proliferation, and survival of tumor B cells [4], by an antigen-driven process that triggers a molecular cascade

These authors contributed equally: Selena Mimmi, Domenico Maisano

These authors jointly supervised this work: Enrico Iaccino, Ileana Quinto

Supplementary information The online version of this article (https:// doi.org/10.1038/s41375-020-0885-y) contains supplementary material, which is available to authorized users.

Selena Mimmi

mimmi@unicz.it

Ileana Quinto

quinto@unicz.it

1 Department of Experimental and Clinical Medicine, "Magna Græcia" University of Catanzaro, Campus "S. Venuta", Viale Europa, Germaneto, 88100 Catanzaro, Italy

2 Department of Clinical Medicine, University "Federico II" of Naples, Naples, Italy of events that lead to transcriptional activation of proliferative and antiapoptotic genes [5-7].

Chronic lymphocytic leukemia (CLL) is a Bproliferative disorder characterized by a clonal expansion and accumulation of neoplastic CD19/CD5/CD23/ CD20-positive B-lymphocytes in blood, bone marrow, and other tissues [8]. Several studies support the hypothesis that a common pool of environmental antigens or self-antigens drives the selection of tumor B cells through the persistent triggering of $\operatorname{IgBCR}[9,10]$. Consistently, the sequence analysis of VDJ rearrangement of tumor IgBCRs revealed a high level of homology in more than $30 \%$ of CLL patients, defined as stereotyped IgBCR, with the prevalence of $\mathrm{VH} 1, \mathrm{VH} 3$, and $\mathrm{VH} 4$ families $[11,12]$. CLL are defined as mutated (M-CLL) or unmutated (U-CLL) depending on the mutational rate of the IgBCR variable regions, being more or less than $2 \%$ respect to the germline, respectively [11, 12]. U-CLL cells expressing the VH1-69 rearrangement usually have an inducible IgBCR and show an aggressive behavior $[11,12]$. In this regard, it would be useful to develop new molecular tools for rapid detection of aggressive CLL clones in peripheral blood.

We previously used phage display for identifying peptide ligands of B-lymphoma, multiple myeloma, and CLL IgBCRs [3, 13-15]. In CLL patients, we documented the co-existence of different CLL clones in a single patient as detected by phage-expressed peptide ligands of the tumor IgBCRs [3, 15]. In this study, we used the phage display for identifying a peptide sequence that was commonly recognized by VH1-69 U-CLL clones of two CLL patients. These patients, named CLL\#1 and CLL\#5, were randomly referred to the Hematology Unit-University Federico II of Naples and initially diagnosed as CLL Binet stage A. At month 8, CLL\#1 worsen to Binet stage $\mathrm{C}$ and returned to Binet stage A after 6 months of 
therapy. CLL\#5 was stably CLL Binet stage A for 2 years of observation. In the course of disease, we analysed four blood samples of patient CLL\#1 (CLL\#1 a-d) and three blood samples of patient CLL\#5 (CLL\#5 a-c). Clinical and laboratory data are reported in Supplementary Table S1. Total RNA was extracted from purified B cells and reverse transcribed in cDNA followed by nested RT-PCR to amplify the heavy and light chains of the Ig variable regions, as previously described [3] (Supplementary Fig. S1). The PCR-amplified products were appropriately digested and cloned in expression vectors for DNA sequencing. The Ig nucleotide sequences were analysed by the international ImMunoGeneTics information system $^{\circledast}$ (IMGT ${ }^{\circledast}$ http://www.imgt.org) in order to find a match with the relative VH and VL families, according to the classification of the stereotyped IgBCRs. In both CLL patients, we observed the co-existence of variable $\mathrm{VH}$ nucleotide sequences, both U-CLL and M-CLL, with a unique VL nucleotide sequence at each time of collection (Table 1) (GenBank accession numbers MT334403 to MT334414).

Among the total CLL populations, an unmutated VH169 CLL subpopulation persisted in the patient CLL\#1 (VH1-69/D3-16*01/J6*02) and patient CLL\#5 (VH1-69/ $\mathrm{D} 7-27 * 01 / \mathrm{J} 3 * 02$ ) all time of observation, ranging between 30 and $80 \%$ of total CLL population. Other CLL clones were either mutated or unmutated, appearing and disappearing over time at a lower percentage (Table 1). In the patient CLL\#1, the VH1-69 clone peaked at $80 \%$ at passage to Binet stage $\mathrm{C}$ and dropped to $30 \%$ after therapy with the remission of disease (Table 1). This evidence indicated that the VH1-69 U-CLL clone had an aggressive behavior as, differently from the other CLL clones, it persisted elevated during the time of observation.

We reasoned that a specific ligand could be a potential tool to discriminate and monitor the VH1-69 U-CLL subpopulation in peripheral blood. To this end, we produced the recombinant CLL IgBCRs, as previously described [3] (Supplementary Fig. S2). The purified VH1-69 U-CLL Ig of patient CLL\#5 was used as bait to screen an M13 phage-displayed random peptide library (RPL) $[3,16]$. The workflow of phage selection is shown in Supplementary Fig. S3. Twenty-five phage colonies were selected and subject to DNA sequencing to determine the amino acid sequence required for the binding to the VH1-69 U-CLL Ig. Three amino acid sequences were identified at different frequencies: p1 (48\% phages), p2 (35\% phages), and p3 (17\% phages) (Fig. 1a). By ELISA, the phage $\mathrm{p} 1$ showed higher affinity binding to VH1-69 U-CLL Ig of patient CLL\#5 compared with phages p2 and p3 (Fig. 1b) (Supplementary Fig. S4). Further, the phage 1 did not bind the other three CLL clones of patient CLL\#5, while phage p2 and p3 did
(Fig. 1b). We then analysed the binding of the three phages to the CLL Igs of patient CLL\#1. The phage $\mathrm{p} 1$ bound only the VH1-69 U-CLL Ig of CLL\#1 with high affinity (Fig. 1b). Differently, the phages p2 and p3 did not bind VH1-69 U-CLL Ig of CLL\#1 and showed a weak binding to other Igs of patient CLL\#1 (Fig. 1b). These results indicated that the phage $\mathrm{p} 1$ exclusively bound the VH1-69 U-CLL IgBCR of both patients CLL\#1 and CLL\#5 (Fig. 1b).

Being the VH1-69 rearrangement of IgBCR often associated with poor prognosis of CLL [12], we considered the possibility to detect the VH1-69 U-CLL population in peripheral blood using phage $\mathrm{p} 1$ as specific ligand. To this end, we analysed the immunophenotype and phage binding profile of $\mathrm{B}$ cells collected from patient CLL\#5 at month 1. Consistently with clinical data (Supplementary Table S1), B cells were 99.5\% CD5positive and expressed the IgM isotype and the lambda light chain (Fig. 1c-e). As control, the phage wild type did not show any binding (Fig. 1f). The phage p1 detected $73.5 \%$ of CD5-positive cells, and the phage $\mathrm{p} 2$ and phage p3 detected $86.0 \%$ and $81.6 \%$, respectively (Fig. 1g-i). These results were consistent with the occurrence of 75\% VH1-69 U-CLL clones in the patient CLL\#5 as detected by VDJ sequence (Table 1) and the specific binding of phage $\mathrm{p} 1$ to the relative VH1-69 UCLL IgBCR (Fig. 1b). The progressive dilution of B-CLL cells with healthy PBMCs caused a parallel decrease of phage p1-positivity, confirming the specific binding of the phage $\mathrm{p} 1$ to the target tumor clones (Fig. 11-p). Thus, the phage $\mathrm{p} 1$ revealed to be a specific probe for detecting the VH1-69 U-CLL population within a mix of blood cells.

CLL harbors different tumor clones in a single CLL patient, which can be identified by the IgBCR structure. Sequencing the tumor IgBCRs determines the variability of tumor clones but is not useful for flow cytometry of tumor clones in real time. To date, the diagnosis of disease is based on the evaluation of total number of CD5-positive cells associated with the stage of lymphoid tissues infiltration. In this context, it would be relevant to have sensitive molecular tools for monitoring the different tumor clones based on IgBCR recognition. This would be possible by developing new reagents for flow cytometry in order to detect and eventually isolate the most aggressive CLL subpopulations for molecular characterization. Our study opens to the possibility to monitor CLL clones in the peripheral blood of patients by using phage ligands as specific probes of CLL subpopulations. In perspective, the validation of this experimental approach on a large number of patients could provide a new method of clusterization of CLL clones based on their epitopic reactivity. 


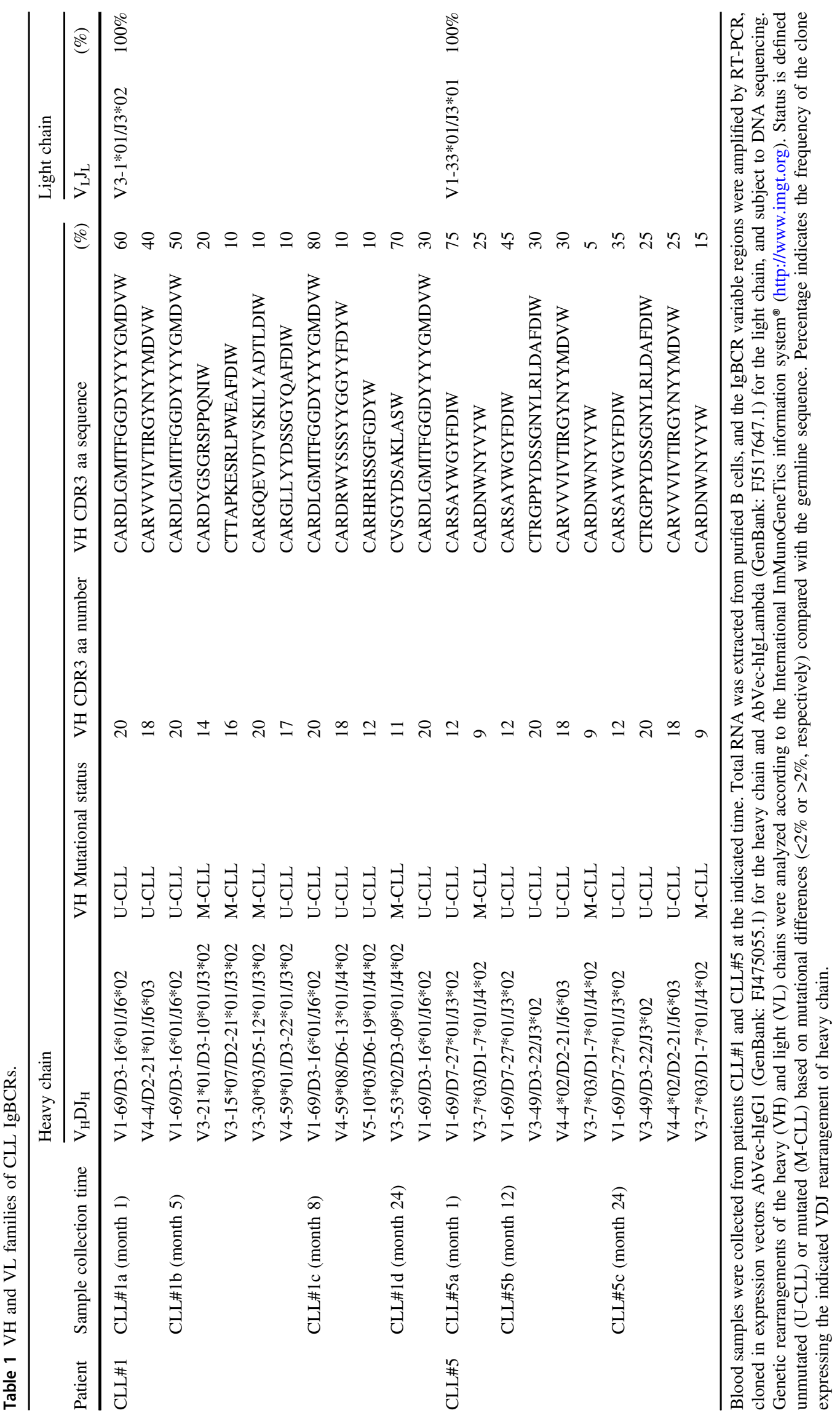


A

\begin{tabular}{|l|l|l|l} 
Peptide name & Sequence (aa) & Freq. $(\%)$ & $\mathbf{K}_{\mathbf{D}}(\mathbf{n M})$ \\
\hline p1 & CRPGQLPSC & 48 & 5,23 \\
\hline p2 & CDYPGQASC & 35 & 10,6 \\
\hline p3 & CQPNRSNSC & 17 & 34,9 \\
\hline
\end{tabular}

B

\begin{tabular}{|c|c|c|c|c|c|}
\hline Patient & IgBCRs clonotypes & p1 & $\mathrm{p}^{2}$ & $\mathrm{p}^{3}$ & wt \\
\hline \multirow[t]{9}{*}{ CLL\#1 } & V1-69/D3-16*01/J6"02 & & & & \\
\hline & $\mathrm{V} 4-4 / \mathrm{D} 2-21^{*} 01 / \mathrm{J} 6^{*} 03$ & & & & \\
\hline & V3-21 $1^{*} 01 / \mathrm{D} 3-10^{*} 01 / \mathrm{J}^{*} 02$ & & & & \\
\hline & $\mathrm{V} 3-15^{*} 07 / \mathrm{D} 2-21^{*} 01 / \mathrm{J}^{*} 02$ & & & & \\
\hline & 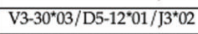 & & & & \\
\hline & $\mathrm{V} 4-59^{*} 01 / \mathrm{D} 3-22^{*} 01 / \mathrm{J}^{*} 02$ & & & & \\
\hline & $\mathrm{V} 4-59^{*} 08 / \mathrm{D} 6-13^{*} 01 / \mathrm{J} 4^{*} 02$ & & & & \\
\hline & $\mathrm{V} 5-10^{*} 03 / \mathrm{D} 6-19^{*} 01 / \mathrm{J} 4^{*} 02$ & & & & \\
\hline & $\mathrm{V} 3-53^{*} 02 / \mathrm{D} 3-09^{*} 01 / \mathrm{J} 4^{*} 02$ & & & & \\
\hline \multirow[t]{4}{*}{ CLL\#5 } & $\mathrm{V} 1-69 / \mathrm{D} 7-27^{*} 01 / \mathrm{J} 3^{*} 02$ & & & & \\
\hline & V3-7*03/D1-7*01/J4*02 & & & & \\
\hline & $\mathrm{V} 3-49 / \mathrm{D} 3-22 / \mathrm{J3}^{*} 02$ & & & & \\
\hline & V4-4. $02 / \mathrm{D} 2-21 / \mathrm{J} 6^{*} 03$ & & & & \\
\hline & & & & & \\
\hline & control lgG1 human & & & & \\
\hline & & & $5<$ fold $<8$ & $3<$ fold $<5$ & $2<$ fold $<3$ \\
\hline
\end{tabular}

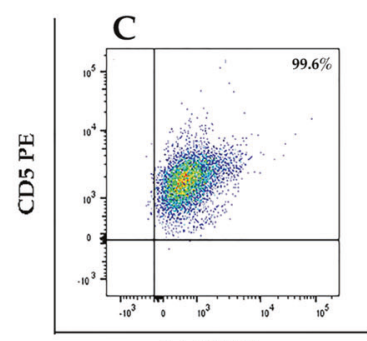

IgM FITC

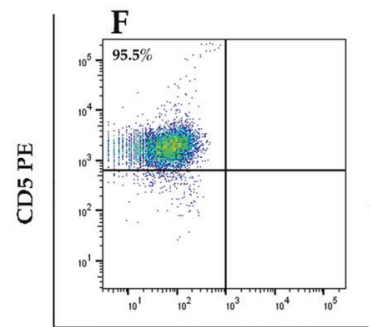

wt phage - FITC

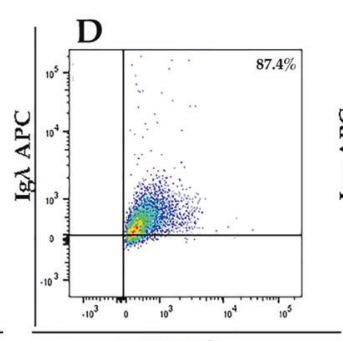

IgM FITC

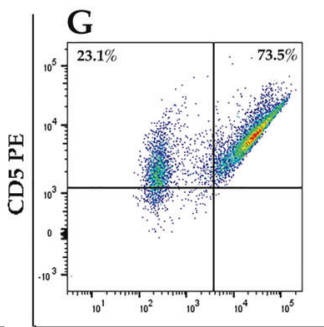

p1 phage - FITC
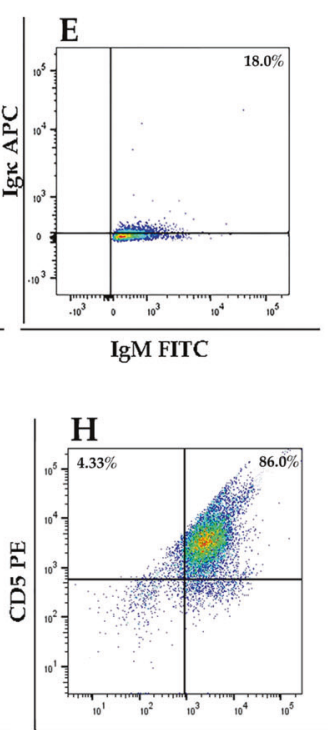

p2 phage - FITC

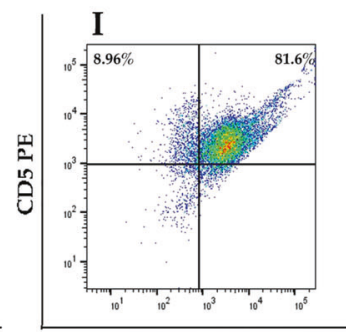

p3 phage - FITC

Serial dilution of CLL\#5a B-CLL cells with healthy PBMCs
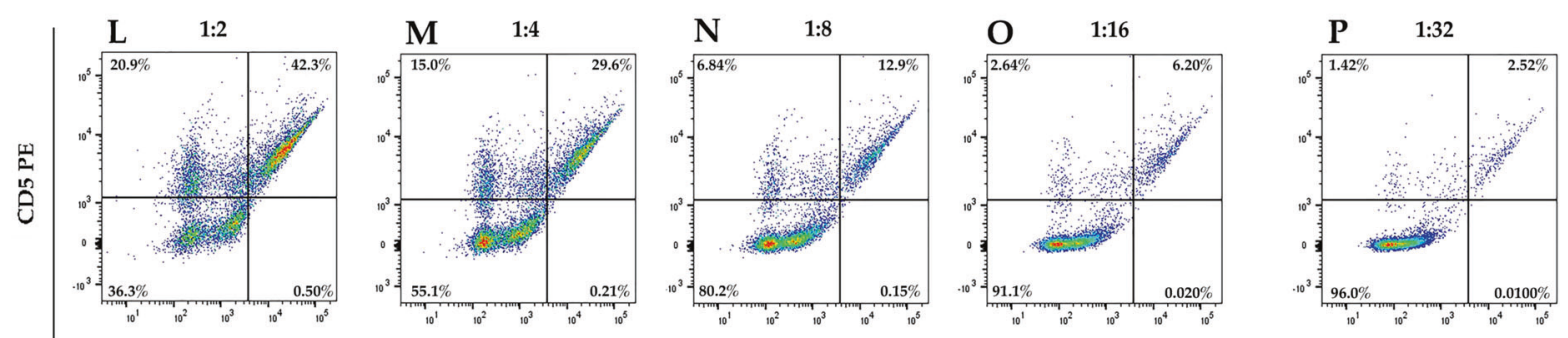

p1 phage - FITC 
Fig. 1 Analysis of phage ligands of CLL IgBCR in patients CLL\#1 and CLL\#5. a Phages ligands of VH1-69 U-CLL IgBCR of CLL\#5 patient. RPL screening was performed using the recombinant VH1-69 UCLL Ig of patient CLL\#5 as bait. The phages p1, p2, and p3 were selected at the indicated frequency; DNA sequencing determined the amino acid (aa) sequence of the insert random peptide. The affinity binding was measured as $K_{D}$ by Scatchard Plot analysis of the experiment shown in Supplementary Fig. S4. b Profile of phage binding to the CLL IgBCRs of patient CLL\#1 and CLL\#5. The recombinant IgBCR $(10 \mathrm{ng} / \mu \mathrm{l})$ was incubated in 96-microwells with the indicated phages $(1 \times$ $\left.10^{9} \mathrm{PFU} / \mu \mathrm{l}\right)$. The phage binding was revealed by the anti-M13 HRP conjugated antibody (Abcam-UK) and relative enzyme substrate. Absorbance was calculated at $405 \mathrm{~nm}$ by the MultiskanTM GO Microplate Spectrophotometer (Thermo Fisher Scientific-USA). Wild-type phage and a human IgG were included as controls. Dark blue corresponds to the highest absorbance ( $>8$-fold respect to the blank); decreasing shades of blue correspond to lower absorbance values, as indicated at the bottom of the table. White squares indicate lack of binding. c-p Phage-based flow cytometry of CLL clones. B cells of patient CLL\#5 collected at month 1 were analyzed by flow cytometry for the expression of CD5, IgM, and the $\operatorname{Ig} \lambda$ and Igא light chains $\mathbf{c}-\mathbf{e}$. The same B-cell sample was analyzed for CD5 expression and phage binding by incubation with the phages wild type (wt) (f), p1 (g), p2 (h), and p3 (i). The B-CLL sample was serially diluted with healthy PBMCs $(1: 2$, 1:4, 1:8, 1:16, 1:32) and analyzed for the binding of phage p1 (1-p). Data were acquired by FACS Canto II (Miltenyi Biotec-Germany) and analyzed by FlowJo Software.

Acknowledgements This work was supported by the following grants: POR FES/FESR 2014-20-ATS ALCMEONE cup J18C17000610006 to IQ; MIUR-PRIN 2017MHJJ55_002 to IQ; GILEAD Fellowship 2018 to EI. SM was supported by funds from the EU project PONAIM1897004-1; DM was supported by funds from the EU project PON-RI2014-2020; EI was supported by the Umberto Veronesi Foundation fellowship.

\section{Compliance with ethical standards}

Conflict of interest The authors declare that they have no conflict of interest.

Ethics statement Experiments involving human subjects were approved by the Italian Regional "Calabria" Ethics Committee (Protocol N. 75, 23/03/17), in accordance with the ethical and safety rules and guidelines provided by the relevant Italian laws (art. 4-5 of D. Igs 116/ 92, DD.MM. of 29/09/1995 and 26/04/2000), and in accordance with the ethical guidelines of the European Community Council (directive n. 86/ 609/ECC). Blood samples from healthy donors or CLL patients were obtained upon written and oral informed consent from the participants to the study.

Publisher's note Springer Nature remains neutral with regard to jurisdictional claims in published maps and institutional affiliations.

Open Access This article is licensed under a Creative Commons Attribution 4.0 International License, which permits use, sharing, adaptation, distribution and reproduction in any medium or format, as long as you give appropriate credit to the original author(s) and the source, provide a link to the Creative Commons license, and indicate if changes were made. The images or other third party material in this article are included in the article's Creative Commons license, unless indicated otherwise in a credit line to the material. If material is not included in the article's Creative Commons license and your intended use is not permitted by statutory regulation or exceeds the permitted use, you will need to obtain permission directly from the copyright holder. To view a copy of this license, visit http://creativecommons. org/licenses/by/4.0/.

\section{References}

1. Chiorazzi N, Efremov D. Chronic lymphocytic leukemia: a tale of one or two signals? Cell Res. 2013;23:182-5.

2. Oppezzo P, Dighiero G. What Do somatic hypermutation and class switch recombination teach us about chronic lymphocytic leukaemia pathogenesis? In: Chronic Lymphocytic Leukemia. Current Topics in Microbiology and Immunology. Berlin, Heidelberg: Springer; 2005. p71-89.

3. Mimmi S, Vecchio E, Iaccino E, Rossi M, Lupia A, Albano F, et al. Evidence of shared epitopic reactivity among independent B-cell clones in chronic lymphocytic leukemia patients. Leukemia. 2016;30:2419-22.

4. Shain K, Tao J. The B-cell receptor orchestrates environmentmediated lymphoma survival and drug resistance in B-cell malignancies. Oncogene. 2014;33:4107-13.

5. Pontoriero M, Fiume G, Vecchio E, de Laurentiis A, Albano F, Iaccino $\mathrm{E}$, et al. Activation of NF- $\mathrm{B}$ in $\mathrm{B}$ cell receptor signaling through Bruton's tyrosine kinase-dependent phosphorylation of IкB- $\alpha$. J Mol Med. 2019;97:675-90.

6. Vecchio E, Golino G, Pisano A, Albano F, Falcone C, Ceglia S, et al. IBTK contributes to B-cell lymphomagenesis in E $\mu$-myc transgenic mice conferring resistance to apoptosis. Cell Death Dis. 2019;10:320.

7. Albano F, Chiurazzi F, Mimmi S, Vecchio E, Pastore A, Cimmino $\mathrm{C}$, et al. The expression of inhibitor of bruton's tyrosine kinase gene is progressively up regulated in the clinical course of chronic lymphocytic leukaemia conferring resistance to apoptosis. Cell Death Dis. 2018;9:13.

8. Scarfò L, Ferreri AJ, Ghia P. Chronic lymphocytic leukaemia. Crit Rev Oncol Hematol. 2016;104:169-82.

9. Stamatopoulos K, Agathangelidis A, Rosenquist R, Ghia P. Antigen receptor stereotypy in chronic lymphocytic leukemia. Leukemia. 2017;31:282-91.

10. Ghia P, Chiorazzi N, Stamatopoulos K. Microenvironmental influences in chronic lymphocytic leukaemia: the role of antigen stimulation. J Intern Med. 2008;264:549-62.

11. Agathangelidis A, Psomopoulos F, Stamatopoulos K. Stereotyped $\mathrm{B}$ cell receptor immunoglobulins in B cell lymphomas. Methods Mol Biol. 2019;1956:139-55.

12. Ten Hacken E, Gounari M, Ghia P, Burger JA. The importance of B cell receptor isotypes and stereotypes in chronic lymphocytic leukemia. Leukemia. 2019;33:287-98.

13. Palmieri C, Falcone C, Iaccino E, Tuccillo FM, Gaspari M, Trimboli $\mathrm{F}$, et al. In vivo targeting and growth inhibition of the A20 murine B-cell lymphoma by an idiotype-specific peptide binder. Blood. 2010;116:226-38.

14. Iaccino E, Mimmi S, Dattilo V, Marino F, Candeloro P, Di Loria A, et al. Monitoring multiple myeloma by idiotype-specific peptide binders of tumor-derived exosomes. Mol Cancer. 2017;16:159.

15. Lupia A, Mimmi S, Iaccino E, Maisano D, Moraca F, Talarico C, et al. Molecular modelling of epitopes recognized by neoplastic B lymphocytes in Chronic Lymphocytic Leukemia. Eur J Med Chem. 2020;185:111838

16. Mimmi S, Maisano D, Quinto I, Iaccino E. Phage display: an overview in context to drug discovery. Trends Pharm Sci. 2019;40:87-91. 